Supporting Information

\title{
Extreme Ultraviolet Radiation Pulsed Atom Probe Tomography of III-Nitride Semiconductor Materials
}

Luis Miaja-Avila $^{1 *}$, Benjamin W. Caplins ${ }^{1}$, Ann N. Chiaramonti ${ }^{1}$, Paul T. Blanchard ${ }^{1}$, Matt D. Brubaker ${ }^{1}$, Albert V. Davydov², David R. Diercks ${ }^{3}$, Brian P. Gorman ${ }^{3}$, Ashwin Rishinaramangalam ${ }^{4}$, Daniel F. Feezell ${ }^{4}$, Kris A. Bertness ${ }^{1}$, and Norman A. Sanford ${ }^{1}$

${ }^{1}$ National Institute of Standards and Technology, Boulder, CO, 80305 USA

${ }^{2}$ National Institute of Standards and Technology, Gaithersburg, MD, 20899 USA

${ }^{3}$ Colorado School of Mines, Golden, CO, 80401 USA

${ }^{4}$ University of New Mexico, Albuquerque, NM, 87131 USA

* Corresponding author: miaja@nist.gov

\section{Sample preparation}

Mg-doped GaN

The Mg-doped layer was approximately $2.2 \mu \mathrm{m}$ thick. The Mg-doped layer was grown at a substrate temperature of $750{ }^{\circ} \mathrm{C}$, with Ga growth rate of $190 \mathrm{~nm} / \mathrm{h}$ and $\mathrm{N}$ flux equivalent to $290 \mathrm{~nm} / \mathrm{h}$ for a V:III ratio of 1.5. The ratio of the $\mathrm{Mg}$ beam equivalent pressure to the $\mathrm{Ga}$ beam equivalent pressure was 0.016 . Hall effect measurements indicated a Hall carrier concentration of $1.1 \pm 0.2 \times 10^{17} \mathrm{~cm}^{-3}$ with a mobility of $14 \pm$ $2 \mathrm{~cm}^{2} \mathrm{~V}^{-1} \mathrm{~s}^{-1}$. SIMS measurements indicated an atomic Mg concentration of $3.0 \pm 0.6 \times 10^{19} \mathrm{~cm}^{-3}$ near the wafer center and $3.5 \pm 0.7 \times 10^{19} \mathrm{~cm}^{-3}$ near the wafer edge. The SIMS measurement also indicated oxygen contamination that compensated approximately half of the holes in the sample.

$\underline{\ln } \times \underline{G} a_{1-x} \underline{N}$

A low temperature $\left(550^{\circ} \mathrm{C}\right) \mathrm{GaN}$ nucleation was grown on c-plane sapphire substrate, followed by a $1 \mu \mathrm{m}$ thick high temperature $\left(\sim 1080^{\circ} \mathrm{C}\right.$ ) GaN buffer. This was followed by $\sim 1.5 \mu \mathrm{m}$ of Si:GaN (doping $\sim 5-6 \mathrm{x}$ $\left.10^{17} \mathrm{~cm}^{-3}\right)$ with the final $300 \mathrm{~nm}$ heavily doped $\left(\sim 3-5 \times 10^{18} \mathrm{~cm}^{-3}\right)$. A $60 \mathrm{~nm}$ of low indium content $\ln _{x} G_{1} a_{1}$ ${ }_{x} \mathrm{~N}(x \approx 0.03)$ underlayer was grown on the $\mathrm{n}-\mathrm{GaN}$ to getter point defects before growing three $\ln _{x} \mathrm{Ga}_{1-\mathrm{x}} \mathrm{N}$ $(x \approx 0.15) / G a N ~ Q W s$ grown at $770{ }^{\circ} \mathrm{C}$. Finally, the device was terminated with a Mg:GaN layer ( $150 \mathrm{~nm}$ doped at $\left.2 \times 10^{19} \mathrm{~cm}^{-3}\right)$, followed by a highly Mg-doped GaN layer $\left(1.2 \times 10^{20} \mathrm{~cm}^{-3}\right)$.

\section{Uncertainty values}

The atomic percentage uncertainty values presented in the main manuscript are solely based on counting statistics due to the Poisson noise in the data. Model uncertainty is another uncertainty source that will be discussed here. We have estimated the model uncertainty by varying the peak ranging limits from $5 \%$ to $50 \%$ of the peak height, changing the peak identification of isobaric peaks, and modifying the background subtraction scheme used. Overall, the model uncertainty in the GaN measurements ranged from $\pm 1-1.5 \%$ for the different specimen measurements. For the Mg-doped GaN measurement it was estimated at $\sim \pm 1.1 \%$. Finally, on the $\mathrm{Al}_{x} \mathrm{Ga}_{1-x} \mathrm{~N}(x \approx 0.5)$ measurement the model uncertainty was $\sim \pm 1 \%$. Unfortunately, due to the low statistics in the $\ln _{x} G_{a}-x N$ QW specimen we were not able to reasonably estimate the model uncertainty. The total uncertainty would be a combination of statistical and model uncertainties.

\section{Segmentation of $\mathrm{GaN}$ and $\mathrm{Al}_{\mathrm{x}} \mathrm{Ga}_{1-\mathrm{x}} \mathrm{N}$ datasets for charge-state-ratio (CSR) analysis:}

In order to use the data to explore the largest range of Ga charge-state ratios (CSRs) possible, it is important to take advantage of known correlations. Two known correlations are between DC voltage and CSR and between the poles on the detector hit map and the CSR. 
The first correlation is straightforward. Plotted in Figure S1 is the standing voltage and CSR for a dataset versus time. Strongly correlated with the discrete increases in the voltage (see main text) is a sudden increase in the observed CSR. Then, the CSR decays as the tip is blunted through evaporation. By averaging the total dataset, we obtain a CSR 0.9, however, segmenting the dataset (based on time) prior to calculating the CSR will permit a range of CSR to be explored. For example, in the dataset shown here the CSR ranges from approximately 0.5 to 2 . For the datasets used in the manuscript the data were split up such that each segment contained approximately $2^{16}$ ions.

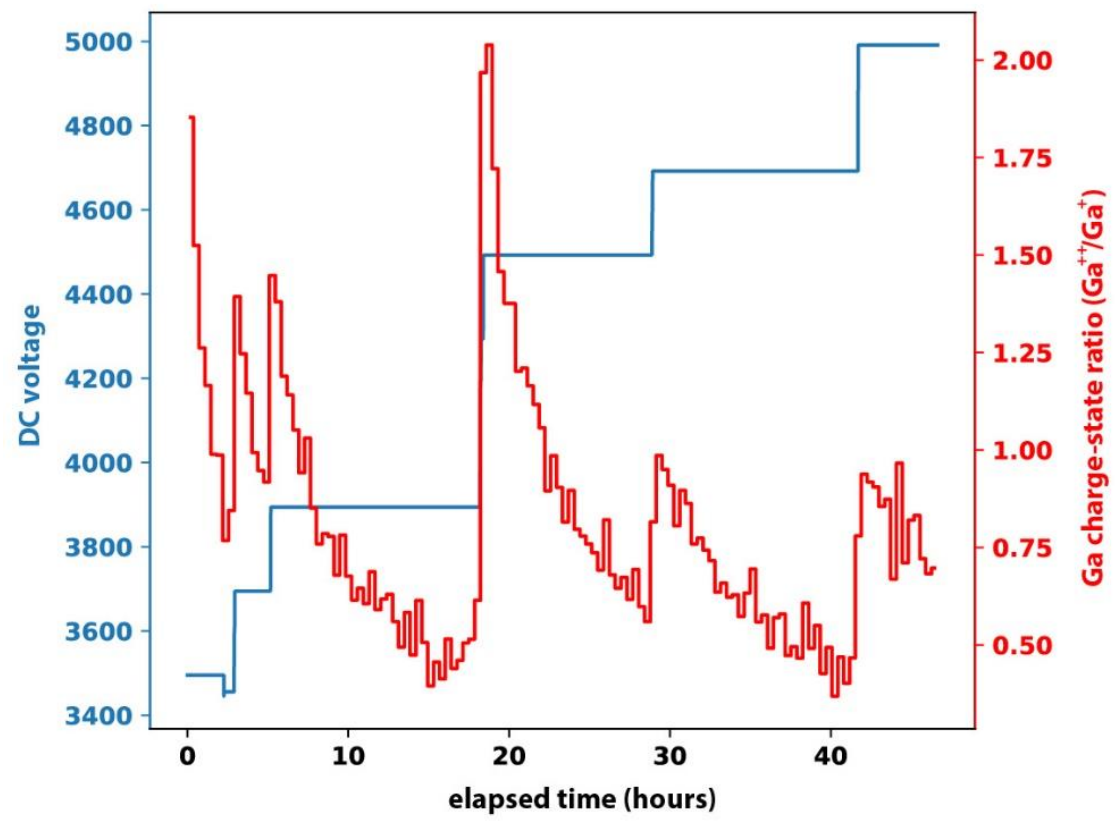

Figure S1. Standing voltage (blue) and Ga charge-state ratio (red) as a function of elapsed time. The changes in Ga charge-state ratio are strongly correlated with modifications of the standing voltage.

The second correlation is between the detector hit map and the CSR and has been well established in the literature ${ }^{1-7}$ In the previous work it was noted that the crystallography has an impact on the evaporation field and ion trajectories in GaN. In many cases, the six-fold symmetric [0001] pole of GaN is visible on the raw hit map and is also clearly visible when the CSR is plotted as a function of detector position. Furthermore, it was demonstrated that the plot of Ga concentration versus CSR generated by changing the DC field is consistent with the plot of Ga concentration versus CSR generated by analyzing annular regions from the detector hit map. ${ }^{6}$ As shown in Figure S2(a), the CSR varies by about an order of magnitude when changing the analysis region on the hit map from the pole center to far from the pole center and it was noted that "any attempt to use the average CSR to perform quantitative composition analysis is inappropriate". ${ }^{6}$ In Figure S2(b) we also plot the total ion hit map and the CSR map. Indicated on the total ion hit map are the same three annular regions that were used to segment the data used in the manuscript. The annular regions were chosen to have equal areas.

Leveraging both correlations allows us to explore a wider range of CSRs than is possible otherwise. In practice, the data are first segmented based on time ( $2^{16}$ ions per segment) then each segment is divided again based on the three annular regions indicated on the hit map. Dividing the data further was not helpful because of poor counting statistics. 


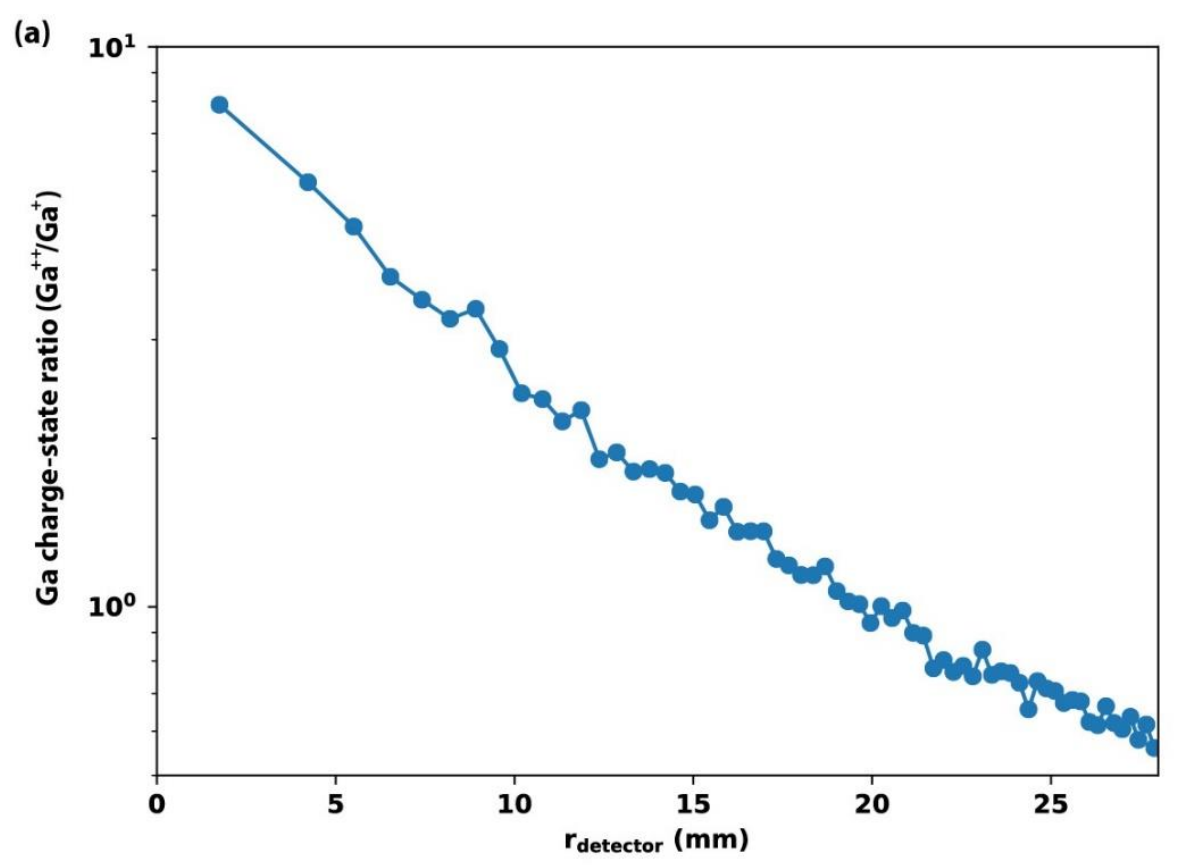

(b)
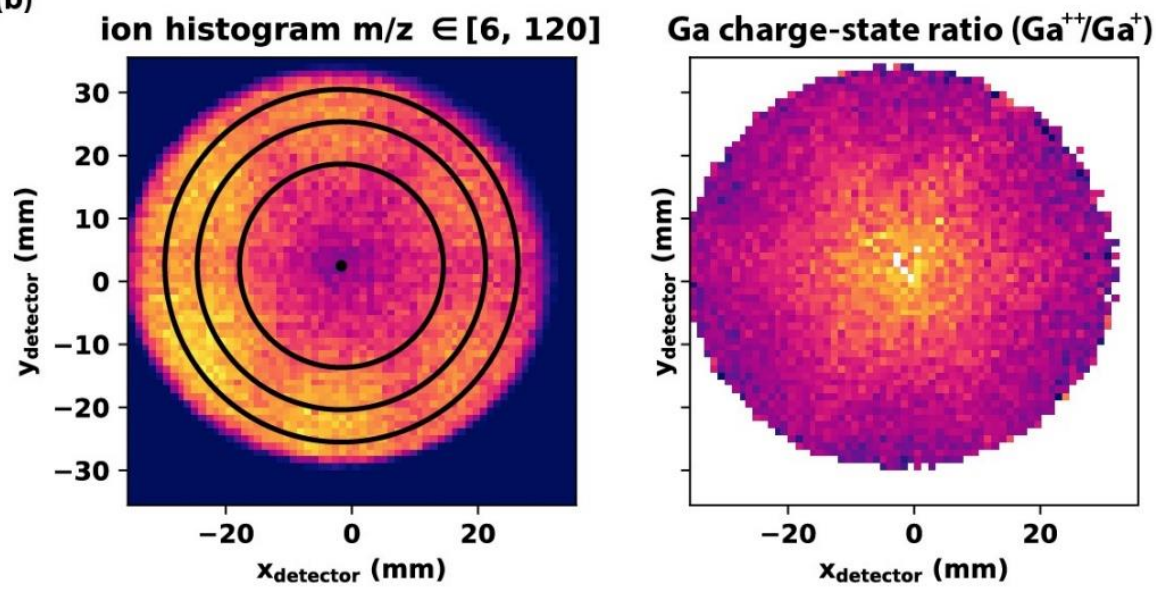

Figure S2. (a) Ga charge-state ratio as a function of radial distance from the center of the detector. (b) lon hit map and Ga charge-state ratio map. Indicated on the ion hit map are the same three annular regions that were used to segment the data used in the manuscript.

As discussed in the manuscript, we performed EUV APT GaN measurements where the target average Ga CSR varied from 0.1 to 1 . Figure S3 shows the mass spectra for each dataset. These datasets are then segmented with the above described method to produce the data presented in Figure 3 of the manuscript. 


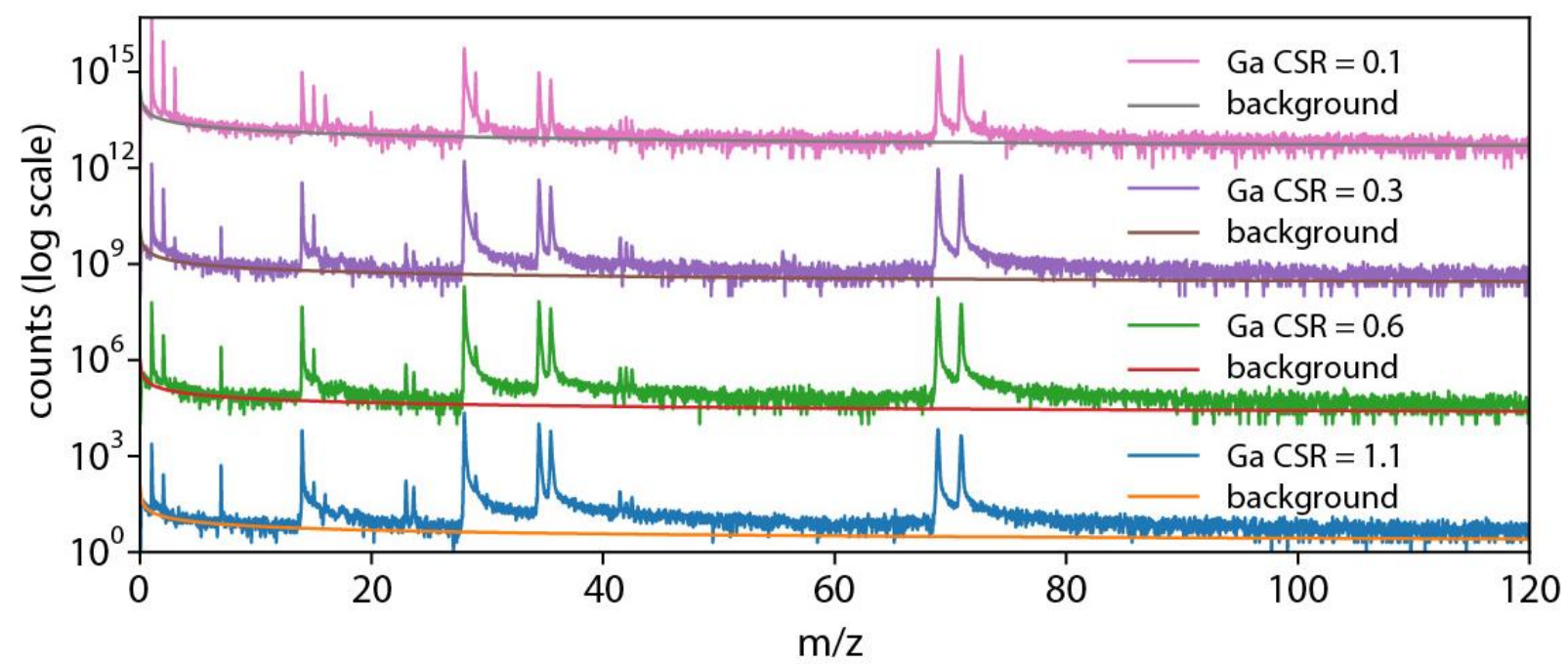

Figure S3. EUV APT mass spectra of GaN (and backgrounds) with various target average Ga CSR. For display purposes, the mass spectra are offset by a factor $10^{4}$.

\section{$\mathrm{Al}_{\mathrm{x}} \mathrm{Ga}_{1-\mathrm{x}} \mathrm{N}$ data analysis}

As discussed in the main manuscript, there is a significant underestimation of $N$ in the $\mathrm{Al}_{x} \mathrm{Ga}_{1-x} \mathrm{~N}$ measurements. Some of the possible processes for this $\mathrm{N}$ underestimation are: N DC evaporation, generation of $\mathrm{N}_{2}$ neutral by dissociation, and detector dead-time effects. (1) A signature of DC evaporation of $\mathrm{N}$ is a drastic increase in the background level as a function of the DC standing voltage. We did not observe this effect in our measurements, so we assume N DC evaporation is not the main reason for our $\mathrm{N}$ underestimation. (2) Generation of $\mathrm{N}_{2}$ neutral species by dissociation has been discussed as a possibility in the literature. Therefore, we plotted the correlation histograms to look for dissociation pathways in our measurement. While we did observe the dissociation pathway for $\mathrm{AlN}^{++} \rightarrow \mathrm{Al}^{+}+\mathrm{N}^{+}$, see Figure S4, we do not see evidence of significant neutral species generation pathways and, therefore, do not believe this is the main cause of our $\mathrm{N}$ underestimation. (3) We analyzed our apparent concentration values as a function of the multiplicity of the detection events, and we observed a significant variation in the $\mathrm{N}$ values between single and multiple detection events. So, it appears possible that a large fraction on $\mathrm{N}$ is under reported in the mass spectrum of $\mathrm{Al}_{x} \mathrm{Ga}_{1-\mathrm{x}} \mathrm{N}$, due to detector dead time events, when performing EUV APT. Further studies are necessary to better understand this $\mathrm{N}$ underestimation. 


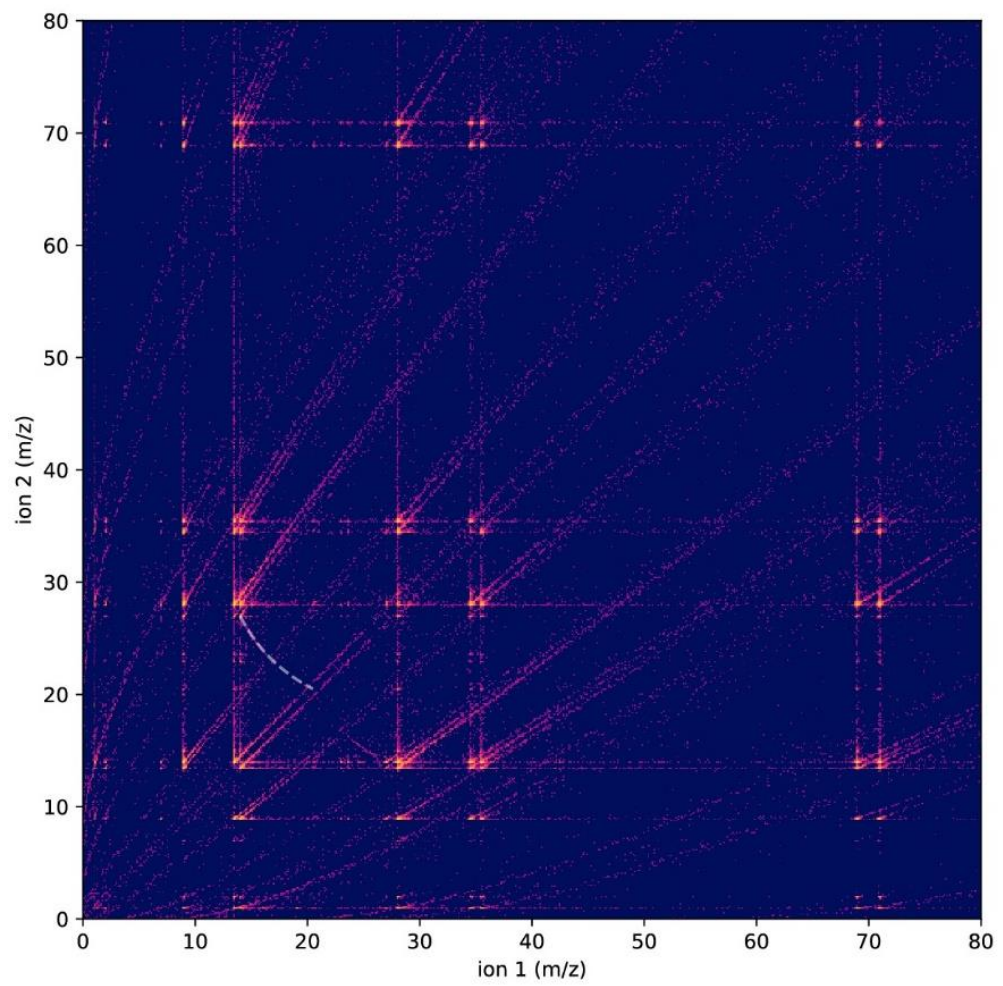

Figure S4. Correlation histogram for EUV APT of $A l_{x} G_{1-x} N(x \approx 0.5)$ in log color scale. The dashed line represents the dissociation pathway for $\mathrm{AlN}^{++} \rightarrow \mathrm{Al}^{+}+\mathrm{N}^{+}$.

\section{$\ln _{\mathrm{x}} \mathrm{Ga}_{1-\mathrm{x}} \mathrm{N}$ results with NUV LAPT}

In order to verify the apparent In concentrations in our EUV APT results from Figure 6, we performed NUV LAPT measurements from the same $\ln _{x} G a_{1-x} N$ sample. This measurement was performed in a LEAP $4000 \mathrm{X} \mathrm{Si}$ atom probe chamber (CAMECA Instruments) ${ }^{*}$ that utilizes a laser operating at a wavelength of $355 \mathrm{~nm}$, pulse duration of $\sim 8 \mathrm{ps}$, and a focused spot size at the sample location of $\sim 2 \mu \mathrm{m}$. The pressure inside the atom probe chamber was $\sim 7 \times 10^{-9} \mathrm{~Pa}\left(5 \times 10^{-11} \mathrm{Torr}\right)$, the sample temperature was $\sim 55 \mathrm{~K}$, and the laser energy per pulse was held constant at $\sim 10 \mathrm{fJ}$, with at $250 \mathrm{kHz}$ repetition rate. The apparent In concentration in the buffer section was $2.4 \pm 0.1$ at. \% and $7.3 \pm 0.4$ at. \% in the quantum well. In comparison, our results using EUV APT show an apparent In concentration of $2.2 \pm 0.1$ at. \% in the buffer section and $7.7 \pm 0.5$ at. $\%$ in the quantum well.

\section{References}

(1) Diercks, D. R.; Gorman, B. P.; Kirchhofer, R.; Sanford, N.; Bertness, K.; Brubaker, M. Atom Probe Tomography Evaporation Behavior of C-Axis GaN Nanowires: Crystallographic, Stoichiometric, and Detection Efficiency Aspects. J. Appl. Phys. 2013, 114, 184903.

(2) Mancini, L.; Amirifar, N.; Shinde, D.; Blum, I.; Gilbert, M.; Vella, A.; Vurpillot, F.; Lefebvre, W.; Lardé, R.; Talbot, E.; et al. Composition of Wide Bandgap Semiconductor Materials and Nanostructures Measured by Atom Probe Tomography and Its Dependence on the Surface Electric Field. J. Phys. Chem. C 2014, 118 (41), 24136-24151.

(3) Rigutti, L.; Bonef, B.; Speck, J.; Tang, F.; Oliver, R. A. Atom Probe Tomography of Nitride Semiconductors. Scr. Mater. 2018, 148, 75-81.

(4) Russo, E. Di; Blum, I.; Houard, J.; Gilbert, M.; Da Costa, G.; Blavette, D.; Rigutti, L. Compositional Accuracy of Atom Probe Tomography Measurements in GaN: Impact of Experimental Parameters 
and Multiple Evaporation Events. Ultramicroscopy 2018, 187, 126-134.

(5) Morris, R. J. H.; Cuduvally, R.; Melkonyan, D.; Fleischmann, C.; Zhao, M.; Arnoldi, L.; van der Heide, P.; Vandervorst, W. Toward Accurate Composition Analysis of GaN and AIGaN Using Atom Probe Tomography. J. Vac. Sci. Technol. B, Nanotechnol. Microelectron. Mater. Process. Meas. Phenom. 2018, 36 (3), 03F130.

(6) Morris, R. J. H.; Cuduvally, R.; Melkonyan, D.; Zhao, M.; Heide, P. Van Der; Vandervorst, W. Atom Probe of GaN/AIGaN Heterostructures: The Role of Electric Field, Sample Crystallography and Laser Excitation on Quantification. Ultramicroscopy 2019, 206 (July).

(7) Di Russo, E.; Cherkashin, N.; Korytov, M.; Nikolaev, A. E.; Sakharov, A. V.; Tsatsulnikov, A. F.; Bonef, B.; Blum, I.; Houard, J.; Da Costa, G.; et al. Compositional Accuracy in Atom Probe Tomography Analyses Performed on III-N Light Emitting Diodes. J. Appl. Phys. 2019, 126 (12).

\footnotetext{
${ }^{*}$ Commercial instruments, equipment, or materials are identified only in order to adequately specify certain procedures. In no case does such an identification imply recommendation or endorsement by NIST, nor does it imply that the products identified are the best available for the purpose.
} 